\title{
One-dimensional rarefactive solitons in electron-hole semiconductor plasmas
}

\author{
Yunliang Wang ${ }^{1}$ and Bengt Eliasson ${ }^{2}$ \\ ${ }^{1}$ Department of Physics, School of Mathematics and Physics, \\ University of Science and Technology Beijing, Beijing 100083, China \\ ${ }^{2}$ SUPA, Physics Department, John Anderson Building, \\ University of Strathclyde, Glasgow G4 ONG, Scotland, UK
}

(Received 09 March 2014; Revised 23 April 2014)

\begin{abstract}
We present a theory for linear and nonlinear excitations in semiconductor quantum plasmas consisting of electrons and holes. The system is governed by two coupled nonlinear Schrödinger equations for the collective wave functions of the electrons and holes, and Poisson's equation for the electrostatic potential. This gives a closed system including the effects of charge separation between the electrons and holes, quantum tunneling, quantum statistic, and exchange-correlation due to electron spin. Three typical semiconductors GaAs, GaSb, and GaN, are studied. For small-amplitude excitations, the dispersion relation reveals the existence of one high-frequency branch due to charge separation effects and one low-frequency branch due to the balance between pressure and inertia of the electrons and holes. For the fully nonlinear excitations, the profiles of quasistationary soliton solutions are obtained numerically, and show depleted electron and hole densities correlated with a localized potential. The simulation results show that the rarefactive solitons are stable and can withstand perturbations and turbulence during a considerable time.
\end{abstract}

PACS numbers: 52.35.Mw, 73.22.Lp, 52.40.Db 


\section{INTRODUCTION}

Modern semiconductor quantum devices, such as spintronics, nanotubes, quantum dots and quantum wells [1,2], work with electrons and holes at nanometer scales [3]. The interaction of intense laser pulses with matter can create electron-hole plasmas at high densities [4], where electrons transit from the valence to the conduction band after absorbing the photon energy by single- or multi-photon absorption with holes created in the valence band. Recently, experiments and simulations have shown the existence of moving bright cavity polariton solitons propagating across the semiconductor microcavity [5, 6]. The formation and properties of bright polariton solitons in semiconductor microcavities operating in the strong-coupling regime are affected by the exciton-photon coupling due to electron-hole pair screening [7]. Experimental observations of acoustic solitons have also been made in a GaAs slab at low temperature with a picosecond acoustic technique [8], and fully developed acoustic solitons have also been observed in several crystalline solids [9]. Quantum dispersion effects associated with electron oscillations have been observed experimentally in metals [10] and in warm dense plasmas [11]. Semiconductors provide a compact and inexpensive medium to observe the quantum dispersion effects. In the miniature semiconductor devices, the quantum effects are very important since the de Broglie thermal wavelength of the charged carriers can be comparable to the characteristic spatial scales of the system [12], and since the electrons and holes are Fermions they obey the Fermi-Dirac statistics. Hence, the effects of quantum tunneling and degeneracy pressure have to be taken into account for electron-hole quantum semiconductor plasmas [13-15]. The presence of quantum effects reduces the threshold electric field for onset of parametric amplification and accordingly pump electric field can be easily achievable in unmagnetized n-type piezoelectric semiconductor [16]. The quantum effects may also lead to quasi-quantum lattices of colloid ions at quantum scales [17].

The formation of solitons is due to a balance between dispersion and nonlinearity. In electron-hole semiconductor quantum plasmas, the dispersion effects are due to charge separation between the electrons and holes and due to quantum recoil, and the nonlinearities are due to the large-amplitude electrostatic potential as well as the quantum degeneracy pressure and the exchange-correlation potential [18-22]. By using quantum hydrodynamic equations [23] for electrons and holes, bright solitons have been investigated theoretically 
for several kinds of semiconductors [24]. It has also been shown that quantum electron oscillation can support the formation of stable dark (rarefactive) solitons and vortices [25] associated with localized positive potentials.

In this paper, we investigate the linear and nonlinear properties of semiconductor plasmas with electron and holes as charge carriers, and in particular the possibility of localized nonlinear structures in the form of solitons. The solitons are characterized by a local depletion of the electron and hole densities, accompanied by a large amplitude localized electrostatic potential, and are formed due to the combined effects of charge separation, quantum tunneling, quantum statistical pressure, and exchange-correlations due to spin.

\section{DYNAMICAL MODEL OF ELECTRON-HOLE SEMICONDUCTOR PLAS-} MAS

Using the translational symmetry in the transverse plane, we here consider the onedimensional propagation in the $x$-direction of nonlinear electrostatic acoustic waves in a semiconductor plasma consisting of equal amounts of electrons and holes. To model the dynamics of the electrons and holes in the semiconductor, we use the nonlinear Schrödinger equation (NLSE) for the electrons and holes,

$$
\begin{gathered}
i \frac{\partial \psi_{e}}{\partial t}+H_{e} \frac{\partial^{2} \psi_{e}}{\partial x^{2}}+\left(\Gamma_{x c e} \phi_{x c e}+\phi-\Gamma_{e}\left|\psi_{e}\right|^{4}\right) \psi_{e}=0, \\
i \frac{\partial \psi_{h}}{\partial t}+H_{h} \frac{\partial^{2} \psi_{h}}{\partial x^{2}}+\left(\Gamma_{x c h} \phi_{x c h}-\phi-\Gamma_{h}\left|\psi_{h}\right|^{4}\right) \psi_{h}=0
\end{gathered}
$$

where $\psi_{e}$ and $\psi_{h}$ are the collective wave functions of the electrons and holes, respectively, normalized by $\sqrt{n_{0}}$, where $n_{0}$ is the equilibrium electron and hole number density. The quantum parameter $H_{e, h}=\hbar^{2} \omega_{p e, p h}^{2} / 2 E_{F e}^{2}$ determines the relative importance of the quantum electron/hole recoil effects, where $\omega_{p e, p h}=\left(4 \pi n_{0} e^{2} / \epsilon m_{e, h}^{*}\right)^{1 / 2}$ is the electron/hole plasma frequency, and $E_{F e, F h}=\hbar^{2}\left(3 \pi^{2} n_{0}\right)^{2 / 3} / 2 m_{e, h}^{*}$ is the electron/hole Fermi energy. Here $\epsilon$ is the relative dielectric constant of the material, $e$ the magnitude of the electron charge, and $m_{e, h}^{*}$ the effective electron/hole mass. The interactions between the electrons and holes are governed by the exchange-correlation and electrostatic (Hartree) potentials. The third term $\Gamma_{x c e, x c h} \phi_{x c e, x c h} \psi_{e, h}$ is due to the electron/hole exchange-correlation potential [18], where $\Gamma_{x c e}=\Gamma_{x c h}=0.985 e^{2} / \epsilon r_{0} E_{F e}$, and $r_{0}=n_{0}^{-1 / 3}$ is the Wigner-Seitz radius. Hence, the normalized potentials are $\phi_{x c e, x c h}=-\left[\left|\psi_{e, h}\right|^{2 / 3}+\alpha_{e, h} \ln \left(1+\beta_{e, h}\left|\psi_{e, h}\right|^{2 / 3}\right)\right]$, where $\alpha_{e, h}=0.034 / a_{B e, B h}^{*}$ 
and $\beta_{e, h}=18.37 a_{B e, B h}^{*}$ with $a_{B e, B h}^{*}=\epsilon \hbar^{2} / m_{e, h}^{*} e^{2} r_{0}$ being the Bohr radius divided by the Wigner-Seitz radius $[18,19,21,22]$. The term $\Gamma_{e, h}\left|\psi_{e, h}\right|^{4} \psi_{e, h}$ originates from the quantum statistical electron/hole Fermi pressure $P_{e, h}=m_{e, h}^{*} V_{F e, F h}^{2} n_{0} / 3\left(n_{e, h} / n_{0}\right)^{3}[19,23,25]$, with $V_{F e, F h}=\left(2 E_{F e, F h} / m_{e, h}^{*}\right)^{1 / 2}$ being the Fermi speed [23], and where the corresponding effective potential is repulsive. Here $\Gamma_{e, h}=E_{F e, F h} / E_{F e}$ is a dimensionless constant and $n_{e, h}=\left|\psi_{e, h}\right|^{2}$ is the electron/hole number density normalized by $n_{0}$. The space and time coordinates are normalized by $\lambda_{D F e}=\left(E_{F e} / 4 \pi e^{2} n_{0}\right)^{1 / 2}$ and $\hbar / E_{F e}$, respectively, and the potential is normalized by $E_{F e} / e$. Equations (1) and (2) are closed by Poisson's equation

$$
\frac{\partial^{2} \phi}{\partial x^{2}}=\left|\psi_{e}\right|^{2}-\left|\psi_{h}\right|^{2}
$$

for the electrostatic potential. Equations (1)-(3) govern the collective electron and hole oscillations due to the charge separation between electrons and holes. We stress that Eqs. (1) and (2) for the dynamics of the electrons and holes include the combined effects of the electron and hole quantum tunneling, the quantum statistical pressures, and exchange and correlation effects due to spin. At short wavelengths, the quantum effects become important and give rise to dispersion effects in the electrostatic wave.

The particular form of Eqs. (1) and (2) are due to the formulation of Manfredi and Haas [23], who derived a collective Schrödinger for an ensemble of electrons. We briefly describe this formalism here. Starting from a multi-stream kinetic model for the electrons, where each stream is governed by a single-particle Schrödinger equation, the continuity and momentum equations are derived by taking moments of the electron distribution function, and using a fluid closure based on equal amplitudes of each stream. Finally, assuming a curl-free electron fluid velocity and employing the ideas similar to the ones of Madelung [27] and Bohm [28], the quantum fluid equations for the electron number density and velocity are transformed to a NLSE for a complex-valued, collective electron wave function. Using the formalism of Ref. [23] for electrons and holes would result in Eqs. (1) and (2) without the exchangecorrelation potential, but including the electrostatic potential $\phi$ and the electron and hole pressure terns proportional to $\left|\psi_{e}\right|^{4}$ and $\left|\psi_{h}\right|^{4}$. The latter are the degeneracy pressures, derived from the second moments of the electron and hole distribution functions, assumed to be purely one-dimensional Fermi-Dirac distributions in the cold limit. (In $D$ dimensions, the pressure terms would instead be proportional to $\left|\psi_{e}\right|^{4 / D}$ and $\left|\psi_{h}\right|^{4 / D}$ [23].) Since the underlying fluid equations have curl-free velocities, vortices can exist in multiple dimensions only 
in the form of point vortices, where the density goes to zero at the center of the vortex [29]. The interaction of vortices in two dimensions exhibit pairing of vortices and stability only for vortices with topological charge (circulation number) equal to \pm 1 [25, 30]. Finally, the exchange-correlation potentials are taken to be in the form given by Brey et al. [18], based on a parametrization suggested by Hedin and Lundqvist [31]. The formalism of the collective Schrödinger equation and application of the exchange-correlation potential for electrons is further discussed in Ref. [19]. In the present model, the electrons and holes are interacting only via the electrostatic (Hartree) potential, while further collective electron-electron and hole-hole interactions take place via the respective exchange-correlation potentials and degeneracy pressures. Hence, the dynamics of electrons and holes in included in our model on an equal footing, which is useful for the investigation of spintronics, nanotubes, quantum dots and quantum wells, where electrons and holes interact on nanometer scales.

In the numerical examples below, we will consider three typical semiconductors $[1,3$, 14, 24], (i) GaAs with parameters $n_{0}=4.7 \times 10^{16} \mathrm{~cm}^{-3}, m_{h}^{*}=0.5 m_{e}, m_{e}^{*}=0.067 m_{e}$, and $\epsilon=12.8$; (ii) GaSb with parameters $n_{0}=1.6 \times 10^{17} \mathrm{~cm}^{-3}, m_{h}^{*}=0.4 m_{e}, m_{e}^{*}=0.047 m_{e}$, and $\epsilon=15.69$; (iii) GaN with parameters $n_{0}=1 \times 10^{20} \mathrm{~cm}^{-3}, m_{h}^{*}=1.3 m_{e}, m_{e}^{*}=0.13 m_{e}$, and $\epsilon=11.3$. For GaAs we then have $H_{e}=9.603, H_{h}=1.287, \lambda_{e}=0.174, \lambda_{h}=0.736, \Gamma_{e}=1$, $\Gamma_{h}=0.134$, and $\Gamma_{x c e}=\Gamma_{x c h}=0.563$, for GaSb we have $H_{e}=4.478, H_{h}=0.526, \lambda_{e}=0.276$, $\lambda_{h}=0.768, \Gamma_{e}=1, \Gamma_{h}=0.117$, and $\Gamma_{x c e}=\Gamma_{x c h}=0.214$, and for GaN we have $H_{e}=1.448$, $H_{h}=0.145, \lambda_{e}=0.761, \lambda_{h}=1.522, \Gamma_{e}=1, \Gamma_{h}=0.100$, and $\Gamma_{x c e}=\Gamma_{x c h}=0.096$.

\section{LINEAR WAVE SPECTRUM}

We first investigate the linear properties of the system (1)-(3). The equilibrium solution of the system (1)-(3) is given by $\psi_{e}=\psi_{e 0}, \psi_{h}=\psi_{h 0}$, and $\phi=0$, where $\psi_{e 0}$ and $\psi_{h 0}$ are complex constants such that $\left|\psi_{e 0}\right|^{2}=\left|\psi_{h 0}\right|^{2}=1$, and where the frequencies are $\Omega_{e 0}=$ $-\left(\Gamma_{x c e} \phi_{x c e 0}-\Gamma_{e}\right)$, and $\Omega_{h 0}=-\left(\Gamma_{x c h} \phi_{x c h 0}-\Gamma_{h}\right)$. The system is linearized by perturbing the equilibrium and setting $\psi_{e}=\left(\psi_{e 0}+\psi_{e 1}\right) \exp \left(-i \Omega_{e 0} t\right), \psi_{h}=\left(\psi_{h 0}+\psi_{h 1}\right) \exp \left(-i \Omega_{h 0} t\right)$, and $\phi=\phi_{1}$, where it is assumed that $\left|\psi_{e 1}\right| \ll\left|\psi_{e 0}\right|$ and $\left|\psi_{h 1}\right| \ll\left|\psi_{h 0}\right|$. The linearized equations are then given by

$$
i \frac{\partial \psi_{e 1}}{\partial t}+H_{e} \frac{\partial^{2} \psi_{e 1}}{\partial x^{2}}-\left[\frac{1}{3} \Gamma_{x c e}\left(1+\frac{\alpha_{e} \beta_{e}}{1+\beta_{e}}\right)+2 \Gamma_{e}\right]\left(\psi_{e 1}+\psi_{e 0}^{2} \psi_{e 1}^{*}\right)+\psi_{e 0} \phi_{1}=0,
$$




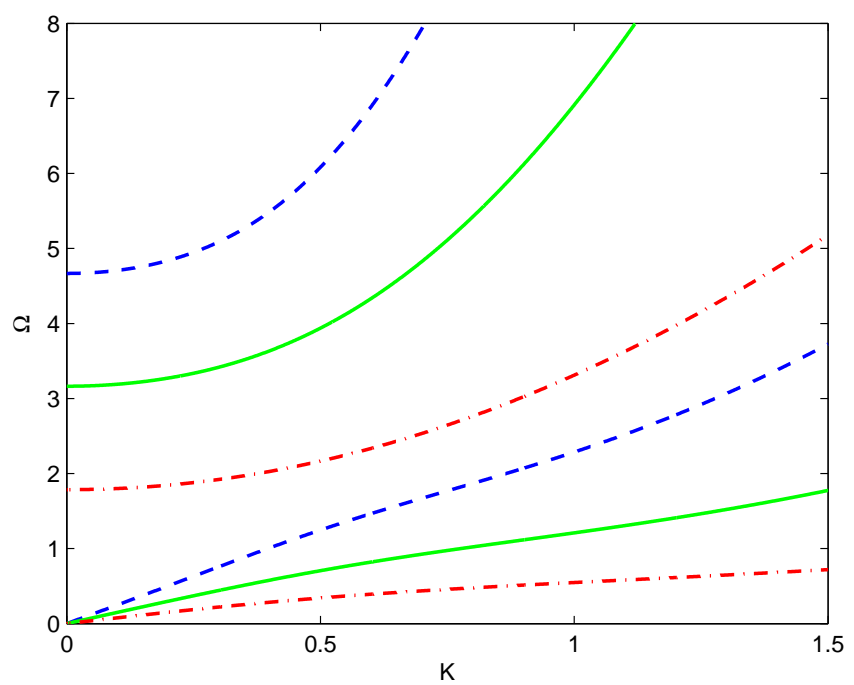

FIG. 1: (Color online) Wave dispersion curves for GaAs (blue dashed line), GaSb (green solid line), and GaN (red dash-dotted line) semiconductor plasmas.

$$
\begin{gathered}
i \frac{\partial \psi_{h 1}}{\partial t}+H_{h} \frac{\partial^{2} \psi_{h 1}}{\partial x^{2}}-\left[\frac{1}{3} \Gamma_{x c h}\left(1+\frac{\alpha_{h} \beta_{h}}{1+\beta_{h}}\right)+2 \Gamma_{h}\right]\left(\psi_{h 1}+\psi_{h 0}^{2} \psi_{h 1}^{*}\right)-\psi_{h 0} \phi_{1}=0 \\
\frac{\partial^{2} \phi_{1}}{\partial x^{2}}=\psi_{e 0} \psi_{e 1}^{*}+\psi_{e 0}^{*} \psi_{e 1}-\left(\psi_{h 0} \psi_{h 1}^{*}+\psi_{h 0}^{*} \psi_{h 1}\right) .
\end{gathered}
$$

Next, the first order quantities are represented by Fourier modes as $\psi_{e 1}=\widehat{\psi}_{e+} \exp (i K x-$ $i \Omega t)+\widehat{\psi}_{e-} \exp (-i K x+i \Omega t), \psi_{h 1}=\widehat{\psi}_{h+} \exp (i K x-i \Omega t)+\widehat{\psi}_{h-} \exp (-i K x+i \Omega t)$, and $\phi_{1}=$ $\widehat{\phi} \exp (i K x-i \Omega t)+\widehat{\phi}^{*} \exp (-i K x+i \Omega t)$, where $\Omega$ and $K$ is the frequency and wave number, respectively, of the electrostatic oscillations. Inserting these Fourier representations into the linearized equations (4)-(6), and separating different Fourier modes and eliminating the Fourier coefficients, we obtain the dispersion relation for the electrostatic oscillations as

$$
1+\frac{1}{D_{L e}}+\frac{1}{D_{L h}}=0
$$

where contributions of the electron and hole oscillations are given, respectively, by

$$
\begin{aligned}
& D_{L e}=-\frac{\Omega^{2}}{2 H_{e}}+\frac{1}{2} H_{e} K^{4}+\frac{1}{3}\left(\frac{C_{x c e}^{2}}{C_{s e}^{2}}+3 \frac{V_{F e}^{2}}{C_{s e}^{2}}\right) K^{2}, \\
& D_{L h}=-\frac{\Omega^{2}}{2 H_{h}}+\frac{1}{2} H_{h} K^{4}+\frac{1}{3}\left(\frac{C_{x c h}^{2}}{C_{s h}^{2}}+3 \frac{V_{F h}^{2}}{C_{s h}^{2}}\right) K^{2},
\end{aligned}
$$

where $C_{x c e}^{2}=0.985\left(1+0.62 / 18.37 a_{B e}^{*}\right) e^{2} / \epsilon r_{0} m_{e}^{*}, C_{x c h}^{2}=0.985\left(1+0.62 / 18.37 a_{B h}^{*}\right) e^{2} / \epsilon r_{0} m_{h}^{*}$, and $C_{s e}^{2}=E_{F e} / m_{e}^{*}$, and $C_{s h}^{2}=E_{F e} / m_{h}^{*}$. We note that the dispersion of the quantum electron oscillation $1+D_{L e}=0$ is identical to that obtained by NLSE-Poisson system in a 
quantum plasma with degenerate electron fluids [26] if the effects of charge exchange and correlation are neglected. We numerically solve the dispersion relation of the semiconductor quantum plasmas, and display the results in Fig. 1. We notice from Fig. 1 that the system supports both high-frequency electrostatic oscillations, similar to plasma oscillations in classical electron-positron or electron-ion plasmas, and low-frequency acoustic-like oscillations. The high-frequency plasma oscillations is primarily due to charge-separation where the electrons and holes oscillate against each other with opposite phases, while the lowfrequency acoustic-like oscillations are due to a balance between pressure and inertia, where the electrons and holes oscillate with the same phases.

\section{THE EXISTENCE AND STABILITY OF QUASISTATIONARY RAREFAC- TIVE SOLITONS}

We next investigate the possibility of nonlinear excitations governed by the system (1)(3). To model nonlinear quasistationary structure moving with a constant speed $v_{0}$, we introduce the ansatz $\psi_{e}=W_{e}(\xi) \exp \left(i K_{e} x-i \Omega_{e} t\right), \psi_{h}=W_{h}(\xi) \exp \left(i K_{h} x-i \Omega t\right)$ and $\phi=\phi(\xi)$ into Eqs. (1)-(3), where $W_{e}$ and $W_{h}$ are real-valued function of the argument $\xi=x-v_{0} t$. The quantities $K_{e, h}$ and $\Omega_{e, h}$ of the electrostatic waves are constant wave number and frequency shifts, respectively. By choosing $K_{e}=v_{0} / 2 H_{e}$ and $K_{h}=v_{0} / 2 H_{h}$, Eqs. (1)-(3) can be written as

$$
\begin{gathered}
\frac{\partial^{2} W_{e}}{\partial \xi^{2}}+\lambda_{e} W_{e}+\frac{1}{H_{e}}\left(\Gamma_{x c e} \phi_{x c e}+\phi-\Gamma_{e} W_{e}^{4}\right) W_{e}=0 \\
\frac{\partial^{2} W_{h}}{\partial \xi^{2}}+\lambda_{h} W_{h}+\frac{1}{H_{h}}\left(\Gamma_{x c h} \phi_{x c h}-\phi-\Gamma_{h} W_{h}^{4}\right) W_{h}=0 \\
\frac{\partial^{2} \phi}{\partial \xi^{2}}=W_{e}^{2}-W_{h}^{2},
\end{gathered}
$$

where $\lambda_{e}=\Omega_{e} / H_{e}-K_{e}^{2}$ and $\lambda_{h}=\Omega_{h} / H_{h}-K_{h}^{2}$ are eigenvalues of the system. From the boundary conditions $\left|W_{e}\right|=1,\left|W_{h}\right|=1$, and $\phi=0$ at $|\xi|=\infty$, we obtain $\lambda_{e}=$ $\Gamma_{e} / H_{e}-\Gamma_{x c e} \phi_{x c e 0} / H_{e}, \lambda_{h}=\Gamma_{h} / H_{h}-\Gamma_{x c h} \phi_{x c h 0} / H_{e}, \Omega_{e}=\Gamma_{e}+v_{0}^{2} / 4 H_{e}-\Gamma_{x c e} \phi_{x c e 0}$ and $\Omega_{h}=\Gamma_{h}+v_{0}^{2} / 4 H_{h}-\Gamma_{x c h} \phi_{x c h 0}$, where the exchange-correlation potential at equilibrium are $\phi_{x c e 0}=-\left[1+\left(0.034 / a_{B e}^{*}\right) \ln \left(1+18.37 a_{B e}^{*}\right)\right]$ and $\phi_{x c h 0}=-\left[1+\left(0.034 / a_{B h}^{*}\right) \ln \left(1+18.37 a_{B h}^{*}\right)\right]$. We solved the system (10)-(12) as a nonlinear boundary value problem with the boundary conditions $W_{e}=-1$ on the left boundary $\xi=-10, W_{e}=+1$ on the right boundary 

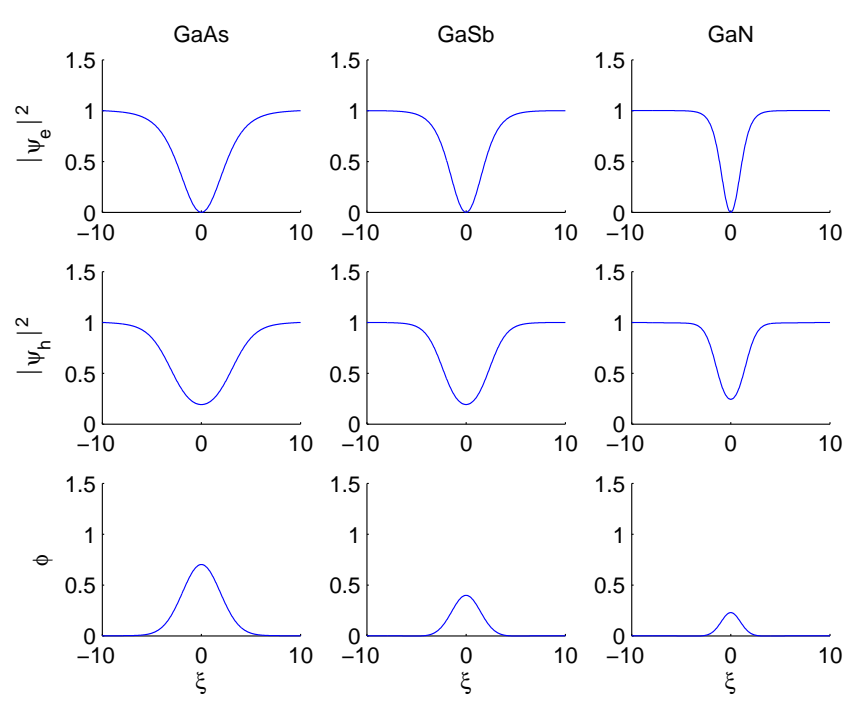

FIG. 2: (Color online) The spatial profiles of the electron number density $n_{e}=\left|\psi_{e}\right|^{2}$ (upper panel), the hole number density $n_{h}=\left|\psi_{h}\right|^{2}$ (middle panel), and the scalar potential $\phi$ (lower panel) for rarefactive solitons in GaAs, GaSb, and GaN semiconductor plasmas (left to right columns, respectively).

$\xi=10$, and $W_{h}=1$ on both the left and right boundary. The potential $\phi$ is set to 0 at the two boundaries. The spatial domain is numerically resolved with 1000 intervals, and the second-derivatives in the system (10)-(12) are approximated by centered second-order approximations. The resulting nonlinear system of equations is then solved numerically by Newton's method. The numerical solutions are displayed in Fig. 2. As seen in Fig. 2, the local depletions of the electron and hole densities are associated with a positive potential. The electron density goes to zero at the center of the solitons, due to the choice of boundary conditions where the electron wavefunction has a phase shift and changes sign at the center of the solitons. The wavefunction of the holes is assumed to the same phase at the left and right boundaries, and since the positively charged holes are repelled by the positive potential, the hole density is also decreased, but not completely depleted, at the center of the solitons.

In order to assess the dynamics and stability of the rarefactive solitons, we have solved the time-dependent system of Eqs. (1)-(3) numerically. We use a pseudospectral method for calculating the spatial derivatives with periodic boundary conditions, and the standard 4th-order Runge-Kutta method to advance the solution in time. The spatial domain is from $x=-5 \pi$ to $x=+15 \pi$ with 512 intervals in space. We do the simulation from 


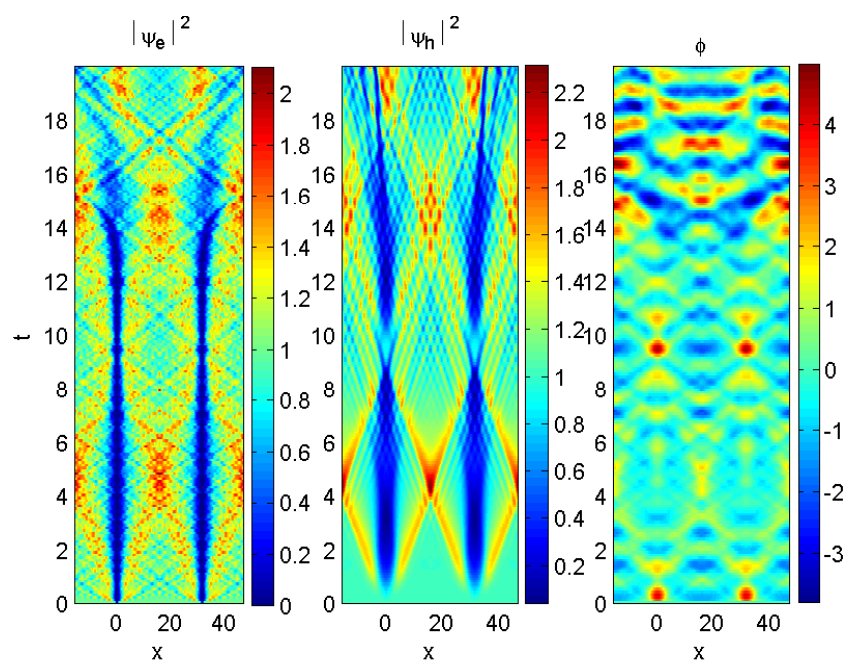

FIG. 3: (Color online) Nonlinear evolution of solitons in a GaAs semiconductor plasma: (a) the electron number density $n_{e}=\left|\psi_{e}\right|^{2}$, (b) the hole number density $n_{h}=\left|\psi_{h}\right|^{2}$, and (c) the scalar potential $\phi$.

time $t=0$ to $t=20$ with time-step being $\Delta t=0.00001$. The initial conditions are $\psi_{e}=\tanh [20 \sin (x / 10)]$ and $\psi_{h}=1$, which is consistent with the periodical boundary conditions used in the simulations. In Figs. 3-5, the space and time evolution of the electron and hole densities are displayed together with the potential, for the GaAs, GaSb and GaN cases, respectively. For the GaAs semiconductor, Figs. 3(a) and (b) show that the soliton withstands perturbations and turbulence during a considerable time. The width of the depletion of $\left|\psi_{e}\right|^{2}$ is larger than that of $\left|\psi_{h}\right|^{2}$, which is consistent with the quasistationary solutions illustrated in Fig. 2. For GaSb, Fig. 4 shows that the soliton also here survives perturbations and turbulence throughout the simulation. The soliton width in the GaSb semiconductor plasma is smaller than that for the GaAs semiconductor, which is also consistent with the quasi-stationary results in left column and middle column in Fig. 2. Finally, for the semiconductor GaN, Fig. 5 shows that the widths of the local depletions of $\left|\psi_{e}\right|^{2}$ and $\left|\psi_{h}\right|^{2}$ in the GaN semiconductor plasma are smaller than for GaAs and GaSb, and the solitons in GaN seem to be more stable than in GaAs and GaSb.

In Figs. 3-5, the simulations show that acoustic-like waves propagate away from the initial soliton profile with a speed that is highest for GaAs and lowest for GaN. The low-frequency dispersion curves in Fig. 1 also confirm that the acoustic speed is highest for GaAs and lowest for GaN. The right columns in Figs. 3-5 also show clearly visible electron-hole plasma 


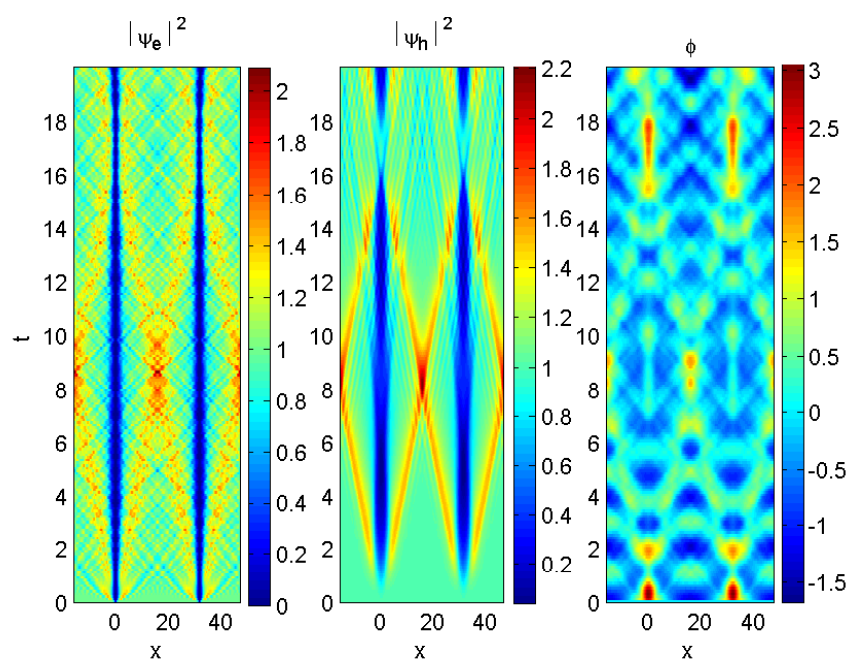

FIG. 4: (Color online) Nonlinear evolution of solitons in a GaSb semiconductor plasma: (a) the electron number density $n_{e}=\left|\psi_{e}\right|^{2}$, (b) the hole number density $n_{h}=\left|\psi_{h}\right|^{2}$, and (c) the scalar potential $\phi$.

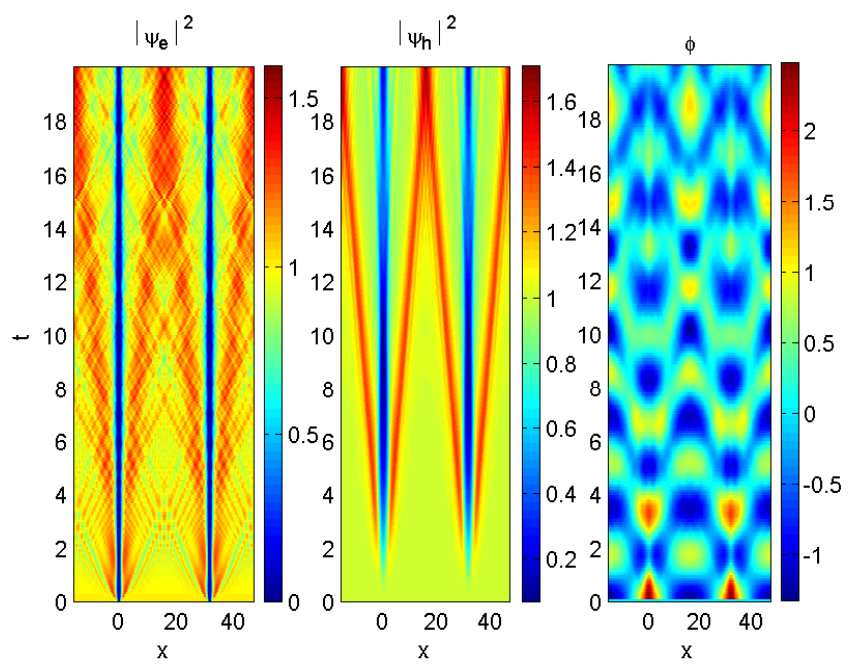

FIG. 5: (Color online) Nonlinear evolution of solitons in a GaN semiconductor plasma: (a) the electron number density $n_{e}=\left|\psi_{e}\right|^{2}$, (b) the hole number density $n_{h}=\left|\psi_{h}\right|^{2}$, and (c) the scalar potential $\phi$.

oscillations in the potential. In the simulations, the plasma oscillations have the highest frequency for the GaAs semiconductor plasma, while the GaN semiconductor has the lowest plasma oscillation frequency. The high-frequency plasma oscillations in the simulations are compatible with the high-frequency dispersion curves in Fig. 1. The amplitude of the localized potential associated with the solitary waves is largest for GaAs and smallest for 
GaN, which is consistent with potential shown in the bottom row in Fig. 2.

\section{SUMMARY AND CONCLUSIONS}

We have investigated the properties of linear and nonlinear quantum electrostatic acoustic waves in an electron-hole semiconductor quantum plasma taking into account the combined effects of the quantum recoil, the degenerate pressure effects, as well as the exchangecorrelation potential due to spin. The dynamics of the system is governed by two coupled NLSEs for the collective wave functions of the electrons and holes, and Poisson's equation for the Hartree potential. The interactions also include the effects of the exchange-correlation potential due to the particle's spin.

By Fourier representations the dispersion relations give two wave modes, the Langmuir mode and the acoustic mode, which shows that the acoustic waves can propagate in semiconductor plasma due to the mass difference between the electrons and holes. A model for quasi-steady-state propagating quantum electrostatic waves is also derived, from which rarefactive solitons were numerically obtained. The numerical simulations show that the solitons are stable and can withstand perturbations and turbulence during a considerable time.

\section{Acknowledgments}

This research is partially supported by NSFC (No. 11104012) and the Fundamental Research Funds for the Central Universities (Nos. FRF-TP-09-019A, FRF-BR-11-031B).

[1] G. Manfredi and P.-A. Hervieux, Appl. Phys. Lett. 91, 061108 (2007).

[2] F. Haas, G. Manfredi, P. K. Shukla, and P.-A. Hervieux, Phys. Rev. B 80, 073301 (2009).

[3] K. Seeger, Semiconductor Physics, 9th ed. (Springer-Verlag, Berlin, 2004).

[4] M. Combescot and J. Bok, J. Lumin. 30, 1 (1985).

[5] A. Amo, D. Sanvitto, F. P. Laussy, D. Ballarini, E. del Valle, M. D. Martin, A. Lemaitre, J. Bloch, D. N. Krizhanovskii, M. S. Skolnick, C. Tejedor, and L. Vina, Nature (London) 457, $291(2009)$. 
[6] O. A. Egorov, D. V. Skryabin, A. V. Yulin, and F. Lederer, Phys. Rev. Lett. 102, 153904 (2009).

[7] O. A. Egorov, D. V. Skryabin, and F. Lederer, Phys. Rev. B 82, 165326 (2010).

[8] E. Péronne and B. Perrin, Ultrasonics 44, e1203 (2006).

[9] H.-Y. Hao and H. J. Maris, Phys. Rev. B 64, 064302 (2001).

[10] H. Watanabe, J. Phys. Soc. Jpn. 11, 112 (1956).

[11] S. H. Glenzer, O. L. Landen, P. Neumayer, R. W. Lee, K. Widmann, S. W. Pollaine, R. J. Wallace, G. Gregori, A. Höll, T. Bornath, R. Thiele, V. Schwarz, W.-D. Kraeft, and R. Redmer, Phys. Rev. Lett. 98, 065002 (2007).

[12] P. K. Shukla and B. Eliasson, Rev. Mod. Phys. 83, 885 (2011).

[13] A. P. Misra, Phys. Rev. E 83, 057401 (2011).

[14] I. Zeba, M. E. Yahia, P. K. Shukla, and W. M. Moslem, Phys. Lett. A 376, 2309 (2012).

[15] M. E. Yahia, I. M. Azzouz, and W. M. Moslem, Appl. Phys. Lett. 103, 082105 (2013).

[16] S. Ghosh, S. Dubey, and R. Vanshpal, Phys. Lett. A 375, 43 (2010).

[17] I. Zeba, Ch. Uzma, M. Jamil, M. Salimullah, and P.K. Shukla, Phys. Plasmas 17, 032105 (2010).

[18] L. Brey, J. Dempsey, N. F. Johnson, and B. I. Halperin, Phys. Rev. B 42, 1240 (1990).

[19] N. Crouseilles, P. -A. Hervieux, and G. Manfredi, Phys. Rev. B 78, 155412 (2008).

[20] Y. Ma, S. Mao, and J. Xue, Phys. Plasmas 18, 102108 (2011).

[21] P. K. Shukla, B. Eliasson, and L. Stenflo, Phys. Rev. E 86, 016403 (2012).

[22] P. K. Shukla and B. Eliasson, Phys. Rev. Lett. 108, 165007 (2012).

[23] G. Manfredi and F. Haas, Phys. Rev. B 64, 075316 (2001)

[24] W. M. Moslem, I. Zeba, and P. K. Shukla, Appl. Phys. Lett. 101, 032106 (2012).

[25] P. K. Shukla and B. Eliasson, Phys. Rev. Lett. 96, 245001 (2006).

[26] D. Pines, Phys. Rev. 92, 626 (1953); D. Bohm and D. Pines, Phys. Rev. 92, 609 (1953).

[27] E. Madelung, Z. Phys. 40, 332 (1926).

[28] D. Bohm, Phys. Rev. 85, 166 (1952).

[29] S. K. Ghosh and B. M. Deb, Phys. Rep. 92, 1 (1982).

[30] I. A. Ivonin, V. P. Pavlenko, and H. Persson, Phys. Rev. E 60, 492 (1999).

[31] L. Hedin and B. I. Lundqvist, J. Phys. C 4, 2064 (1971). 